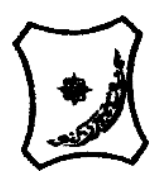

Bayero Journal of Pure and Applied Sciences, 14(1): 45 - 53

Received: March, 2021

Accepted: May, 2021

ISSN $2006-6996$

\title{
EXPERIMENTAL ASSESSMENT ON PREPARATION OF BIODEGRADABLE POLYTHYLENE/POLYLATIC ACID-CHITHOSAN COMPOSITE FROM RENEWABLE RESOURCES
}

\author{
${ }^{1}$ Gumel, B.U., ${ }^{1}$ Gumel, S.M., ${ }^{2}$ Bawa, A.A. and ${ }^{1}$ Auwal, A.S. \\ ${ }^{1}$ Department of Pure and Industrial Chemistry, College of Nation and Pharmaceutical Sciences, Bayero \\ University Kano. \\ ${ }^{2}$ College of education and preliminary studies, Kano P.M.B 3511, Kano State \\ Corresponding author email" bashigume69@gmail.com 08066606828
}

\section{ABSTRACT}

Due to poor degradability and contamination risk of synthetic plastics, utilization of renewable resources is encouraged. Biobased thermoplastic polymers from renewable resource that is inexpensive, biodegradable, compostable and renewably non-toxic, is focused. In this paper mixtures of synthetic and natural polymers were used as a potential option to reduce pollution by plastic waste. The study is aimed at assessing utilization of sweet potato waste as a source of bioplastic for package application, the polymer was modified with a biopolymer chitosan to obtain polylactic acid-chitosan plastic. The developed polymer matrix was blended with polyethylene to obtain biodegradable packaging material. The bioplastic was characterized using fourier Transformed Infra-Red Spectroscopy (FTIR) and scaning electron microscope (SEM). Physical and mechanical properties of the composites were evaluated by measuring enzymatic degradation, tensile strength, and elongation at break. The results have shown that the film obtained have homogeneous surface by (SEM). Mechanical properties of the bio plastics revealed that tensile strength increases with increases in the concentration of chitosan and hence, the elongation at break decreases with increase in chitosan content. While the fastest enzymatic degradation was observed to have high microbial growth on the bio plastics with high content of Chitosan-Polylactic acid.

KEY WORDS

Polyethylene, sweet potato waste, chitosan, poly lactic acid, composite.

\section{INTRODUCTION}

Petroleum based plastics such as polyethylene (PE) polyvinyl chloride (PVC), polypropylene $(\mathrm{PP})$, polyethyleneterephthalate (PET) poly styrene (P.S) and polyamide (P.A) have been widely used as packaging materials due to their availability, flexibility and affordability (Akbar etal.,2019). However, large production and utilization of those synthetic polymers led to the accumulation of plastics wastes that create a serious environmental threat due to their poor biodegradability. Hence the need for plastics from renewable sources which are biodegradable and non-toxic (Formin etal.,2017).

Biodegradable plastics are produced from biopolymers obtained from biomass, such as starch, cellulose, or proteins. Among those biopolymers, starch would be suitable for the manufacture of bioplastics, because it is renewable, biodegradable, inexpensive and compostable (Akbar et al., 2019)

However, starch-based plastics have shown some drawn back, such as brittleness, high sensitivity to moisture and poor mechanical strength compared to polyethylene (Akbar et al., 2019).

Blends of synthetic and natural polymers can yield a new class of materials with improved biodegradability and mechanical strength that can alternatively contribute to the reduction of environmental damages caused by plastic wastes (Formin et al., 2017)

Sweet potato is one of the sources for starch, because it contains $(50-80 \%)$ starch on a dry basis and the starch comprises of $(70-80 \%)$ of highly branched amylopectin and $(20-30 \%)$ of linear and slightly branched amylose (Akbar etal.,2019)

Chitosan is amino polysaccharide cationic polymer that is obtained from chitin by alkaline deacetylation. Chitosan is one of the polysaccharide commonly found in nature and it's film have great potential to be used as packaging materials due to it's antimicrobial activity, non-toxicity and biodegradability (Ke-ke etal.,2007). 
BAJOPAS Volume 14 Number 1, June, 2021

Polylactic acid is a highly versatile and biodegradable aliphatic polyester derived from $100 \%$ renewable resources such as corn, potato, sugar caneetc (Layla et al., 2018). Polylactic acid is a thermoplastic that offers great promise in a wide range of commodity applications (Mohd etal., 2015). Chitosan is a rigid material that can be used to modify the thermal, plastic and antimicrobial properties of polylactie acid without affecting it's biodegradability.

Thus, polylactic acid can be modified with chitosan by amidation reaction between the amino group of the main chain of chitosan and the terminal carboxyl group of the poly lactic acid Nurshahida and Zainoha (2015)

However, the co-polymer blends of chitosanpolylactic shows some deficiencies in used as a substitute for packaging application, mechanical properties, such as tensile strength, elongation to break and thermal stability. Hence, to improve these properties polyethylene (low density polyethylene) can be used as a composite with the co-polymer blends as reported by Vincento (2013)

The aim of this study, is to prepare film based on blends of synthetic polymers with natural polymers, so as to have biodegradable, biocompatible, and ecofriendly packaging materials, using simple hand lay-up technique.

Hand lay-up method is the oldest and most widely used method with acceptable mechanical properties and environmental friendly Tarek and Bader (2016).

\section{MATERIAL AND METHODS}

Materials: Sweet potato waste used for the synthesis of polylactic acid was obtained from Yankaba Market Nassarawa Local Government Area of Kano state; Aspergilus niger and potato dextrose agar (loxoid UK), were obtained from Department of Microbiology, Bayero University Kano;

Short harmed grasshopper used for the preparation of chitosan was obtained from Maigatari international market, Jigawa State, Nigeria.

All other chemicals, like, Hydrochloric acid, (Aldrich), Acetic acid, glycerol (Aldrich), Sodium hydroxide were analytical reagents grades obtained from analytical laboratory, Bayero University, Kano.

\section{METHODS:}

$>$ Starch extraction and preparation of poly(lactic acid)

The potato feel (waste) was founded using mortar and pestol to obtained fine powder. The potato waste powder was dissolved in distilled water with solid to liquor ratio of $(1: 2, w \mid v)$. The mixture was filtered with Muslin cloth. The starch was separated from the liquid by decantation. The starch was collected and oven dried at $50^{\circ} \mathrm{C}$ for 24 hours. The dried powder was kept in an air tight container (Suryani et al., 2017)

Extraction of chitosan from the exoskeleton of grasshopper

Chitosan was extracted from the exoskeleton of short horned grasshopper by chemical treatments.

$150 \mathrm{~g}$ of the exoskeleton of grasshopper was treated with $5 \% \mathrm{HCl}$ to remove the mineral content (deminierilization process) at $30^{\circ} \mathrm{C}$ for $1 \mathrm{~h}$ with solid to liquid ratio, $(1: 15 \mathrm{w} / \mathrm{v})$. The mixture was washed in a running tap water and then rinsed 3 times with distilled water to neutrality. The pasty matter was then treated with $3 \% \mathrm{NaOH}$ at $30^{\circ} \mathrm{C}$ for 1 hour with material to liquid ratio (1-50) to remove the protein content (deprotienization). The resultant chitin was then treated with $50 \% \mathrm{NaOH}$ solution at $120^{\circ} \mathrm{C}$ for $2 \mathrm{hrs}$ with solid to liquor ratio of $(1: 50 \mathrm{w} / \mathrm{v})$. The mixture was stirred periodically for homogeneity. The resultant chitosan was washed to neutrality and oven dried at $50^{\circ} \mathrm{C}$ for 24hrs (Yaret et al.,2018).

The chitosan was then purified by dissolving in $2 \%$ acetic acid for $30 \mathrm{~min}$. at $60^{\circ} \mathrm{C}$ under constant stirring and then re- precipitated using $20 \% \mathrm{NaOH}$ with solid to liquid ratio $(1: 20 w / v)$. The pure chitosan was then washed to neutrality and oven dried at $50^{\circ} \mathrm{C}$ for $24 \mathrm{hrs}$. The pure chitosan was then milled into fine powder with an electrical blender and kept in an airtight container. 
Exoskeleton of short-horned grasshopper $+5 \% \mathrm{HCl}$
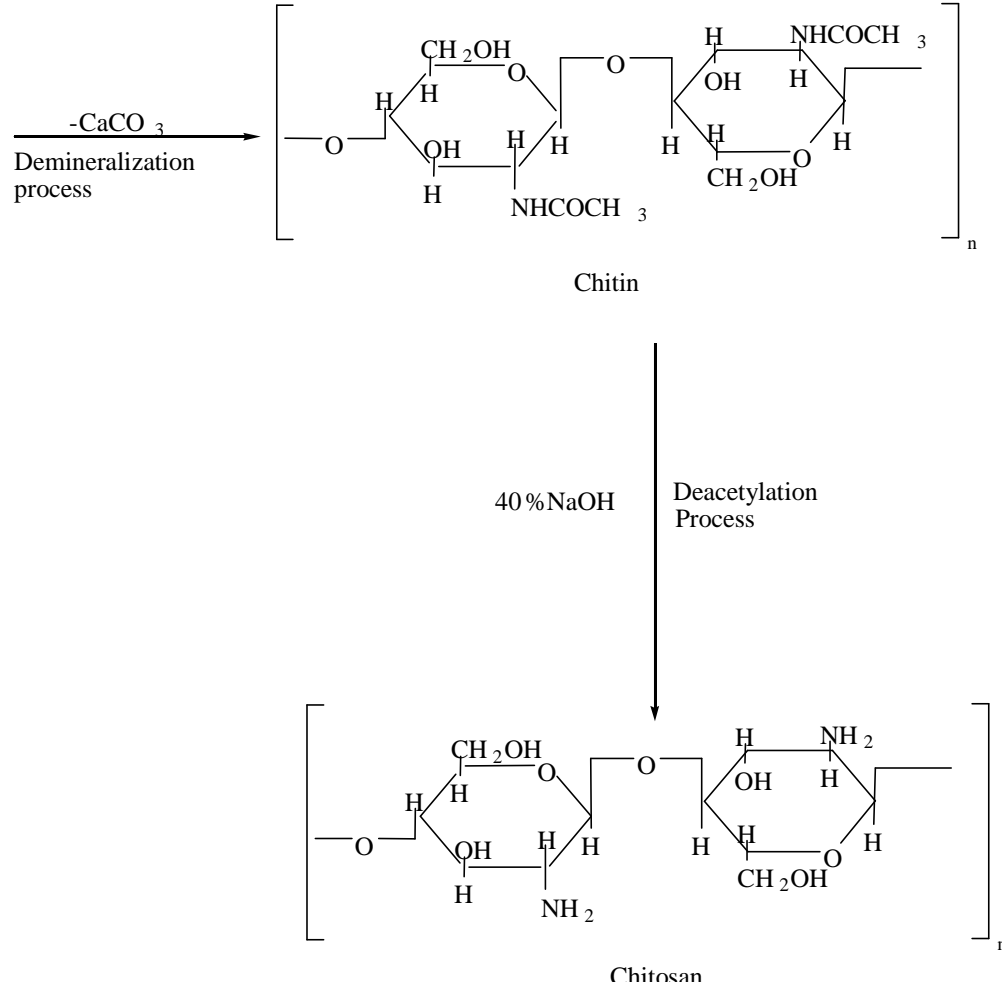

Scheme 1:Schematic representation of chitosan synthesis, Karim., (2018)

\section{Synthesis of Polylatic Acid}

$100 \mathrm{~g}$ of the potato starch was hydrolyzed by the addition of $5 \% \mathrm{HCl}$. The mixture was heated on a hot plate at constant temperature of $60^{\circ} \mathrm{C}$ with constant stirring for 3hours. The glucose was then inoculated with Lactobacillus bulgaricus and incubated in an orbital shaker for 3-days to produce lactic acid (Akbar et al., 2019) The lactic acid was then polymerized to form polylactic acid.

Potato Waste $\longrightarrow$ Strac Starch $\stackrel{\text { hydrolysis }}{\longrightarrow}$ Lactic Acid $\stackrel{\text { Polymerization }}{-\mathrm{H}_{2} \mathrm{O}}$ Lactide
Ring Opening

[lin, et al., 2012]

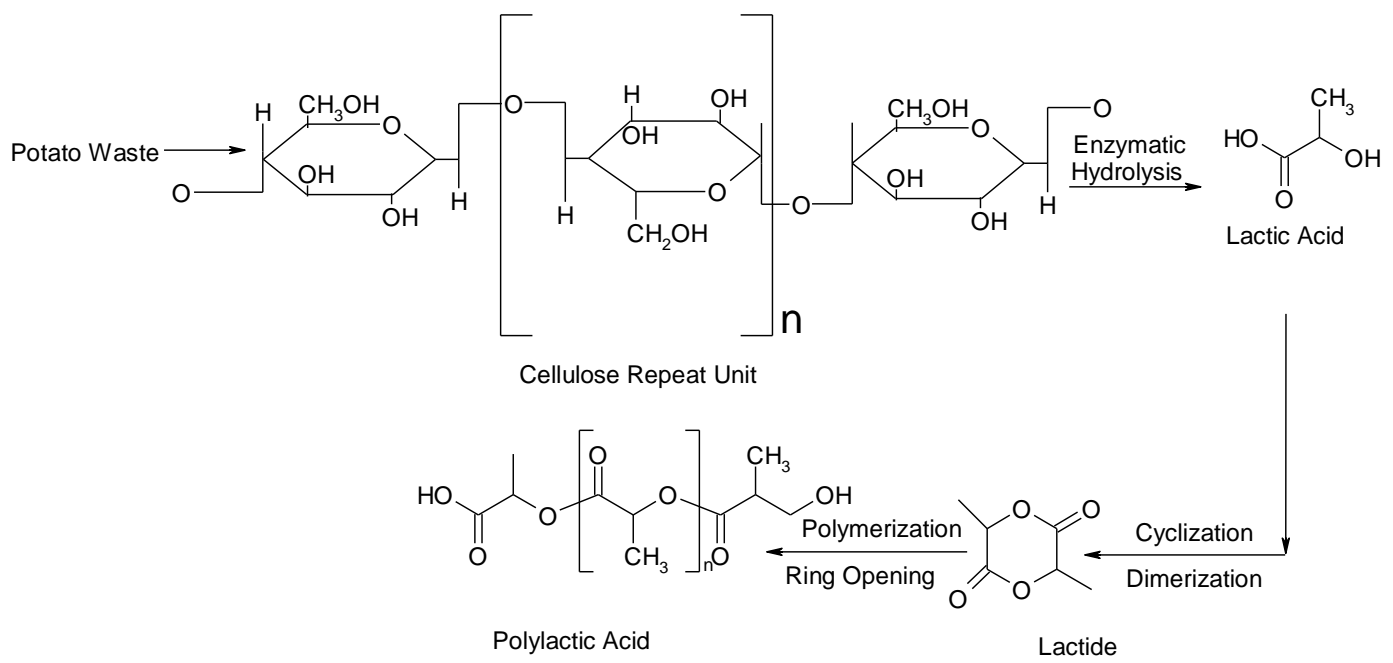

Scheme 2: Schematic representation of polylactic acid synthesis 
BAJOPAS Volume 14 Number 1, June, 2021

\section{$>$ Modification of polylactic acid with chitosan}

Poly (lactic acid) modification was carried out by amidation reaction between the amino groups, in the glucosamine unit of chitosan and the terminal carboxyl group of polylactic acids chains (Suryani et al., 2017). $2 \mathrm{~g}$ of the chitosan powder was added to the polylactic acid and the mixture was dissolved in $200 \mathrm{ml}$ of THF.

The two solutions were mixed and stirred at constant rate of $350 \mathrm{rpm}$ at $30^{\circ} \mathrm{C}$ for $3 \mathrm{hrs}$ (Formin etal., 2017) The modified polylactic acids was purified by dialysis in deionized water for 48hours with water changes at 6 hrs interval, after which the polylactic acids were recovered and freeze dried for $48 \mathrm{hrs}$ under vacuum atmosphere of $0.5 \mathrm{mBar}$ at $-46^{\circ} \mathrm{C}$ in the collector (Layla et al., 2018).

The process was repeated using $4 \mathrm{~g} \& 6 \mathrm{~g}$ of chitosan respectively

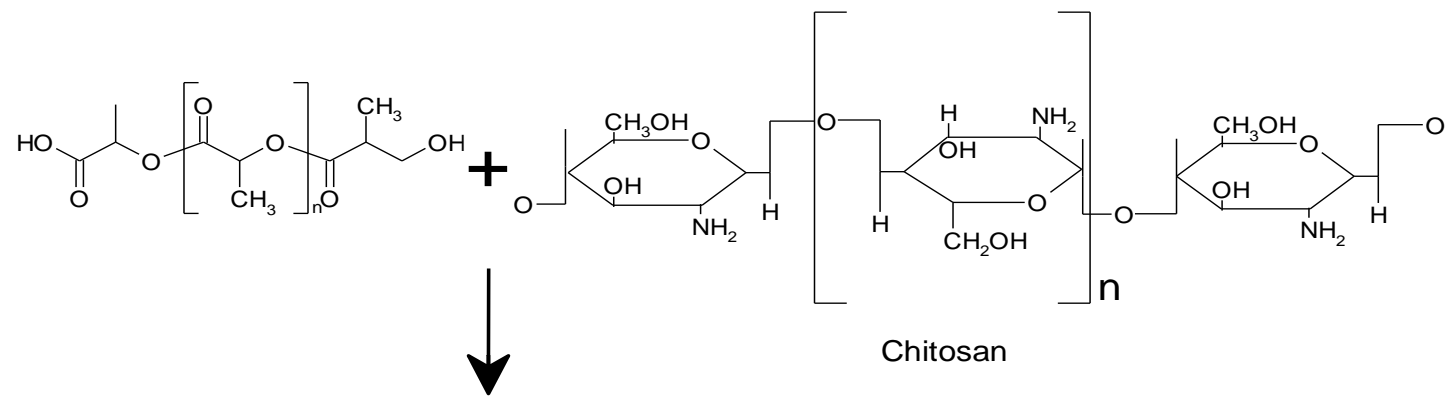

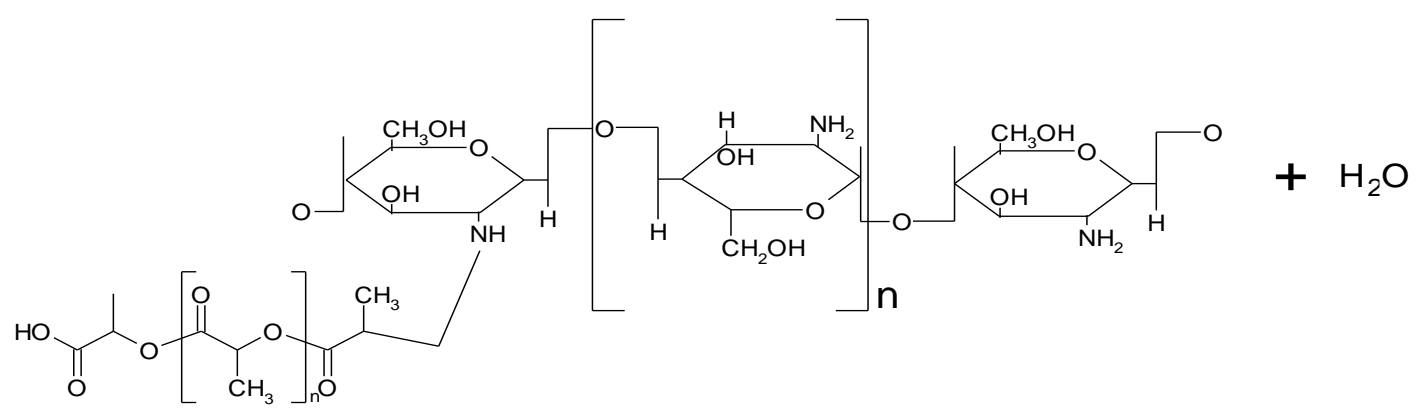

Scheme 3: Schematic representation of modification of polylactic acid with chitosan

Synthesis of films of polyethylene and chitosan modified polylactic acid nano composites. The film of polyethylene and chitosan modified polylactic acid was prepared using $5 \%, 10 \%$ and $15 \%$ polyethylene (PE).

The polymer blends each for polyethylene with Ch-PLA were subjected to mechanical agitation for 30 minutes until a homogeneous mixture was obtained. The films were then poured in mould and oven dried at $50^{\circ} \mathrm{C}$ for $72 \mathrm{hrs}$.

The films were characterized using FTIR, SEM and mechanical properties using tensile test equipment, universal testing machine (model exceed E. 43, Japan) and Enzymatic degradation to assess biodegradability (Karim, 2018).

\section{RESULTS AND DISCUSSION}

\section{FTIR SPECTRUM}

The FTIR spectrum of Chitosan, chitosan modified polylactic acid displayed the following absorption characteristics shown table 1 :

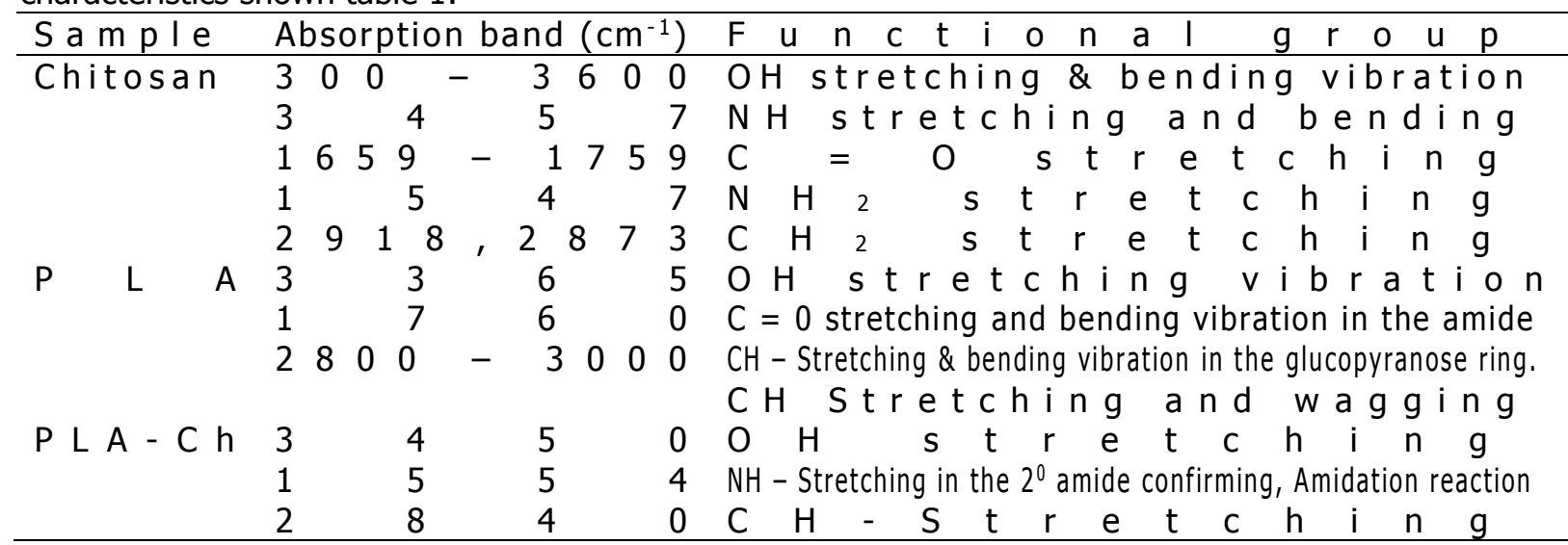




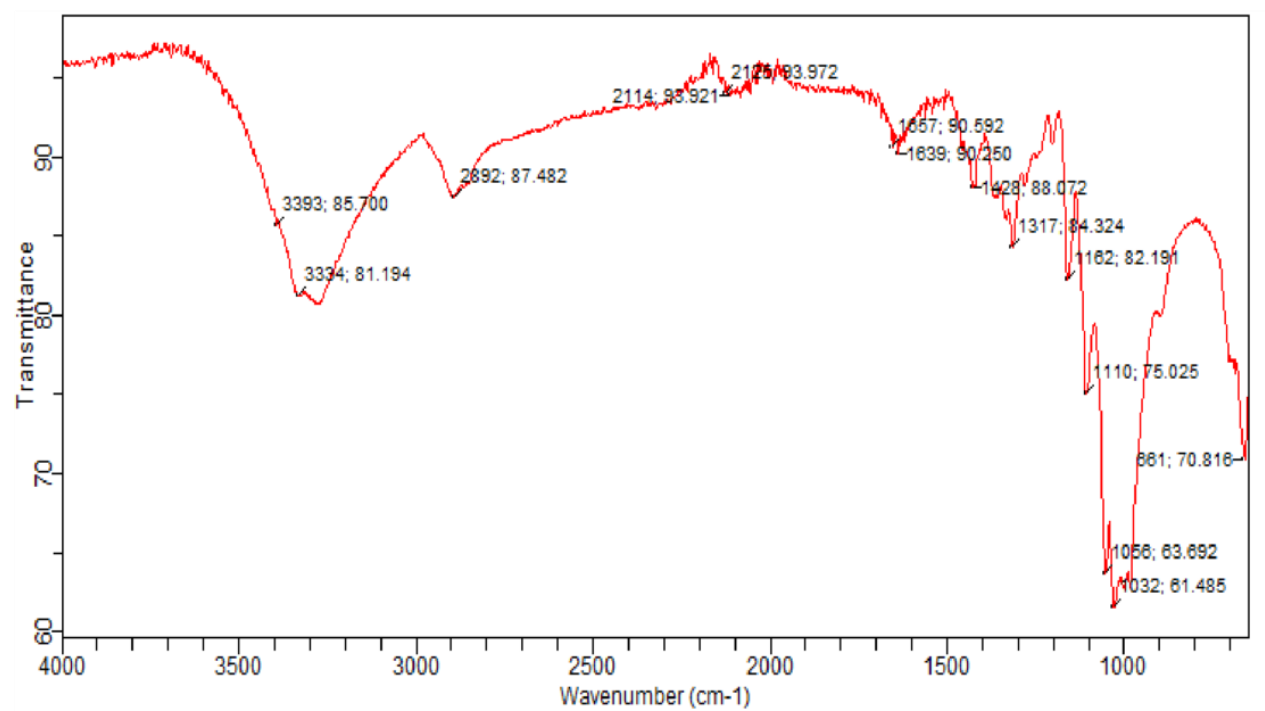

Figure 1a:FTIR spectrum ofpolylactic acid

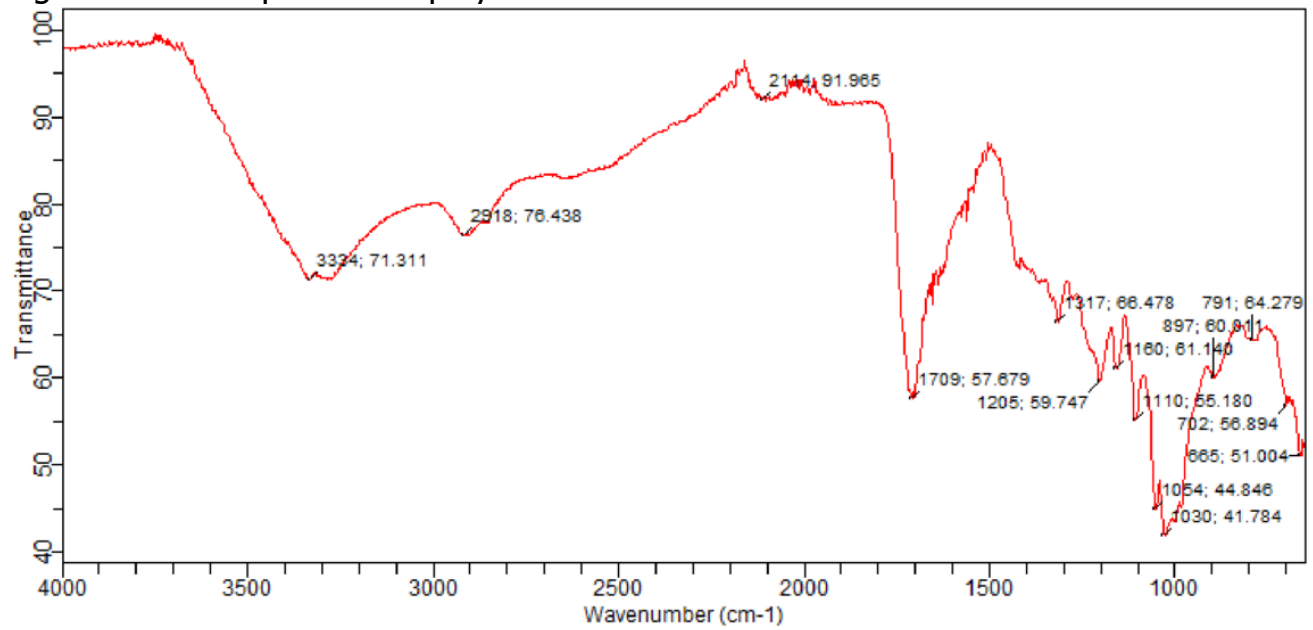

Figure 1b: FTIR Spectrum of Chitosan

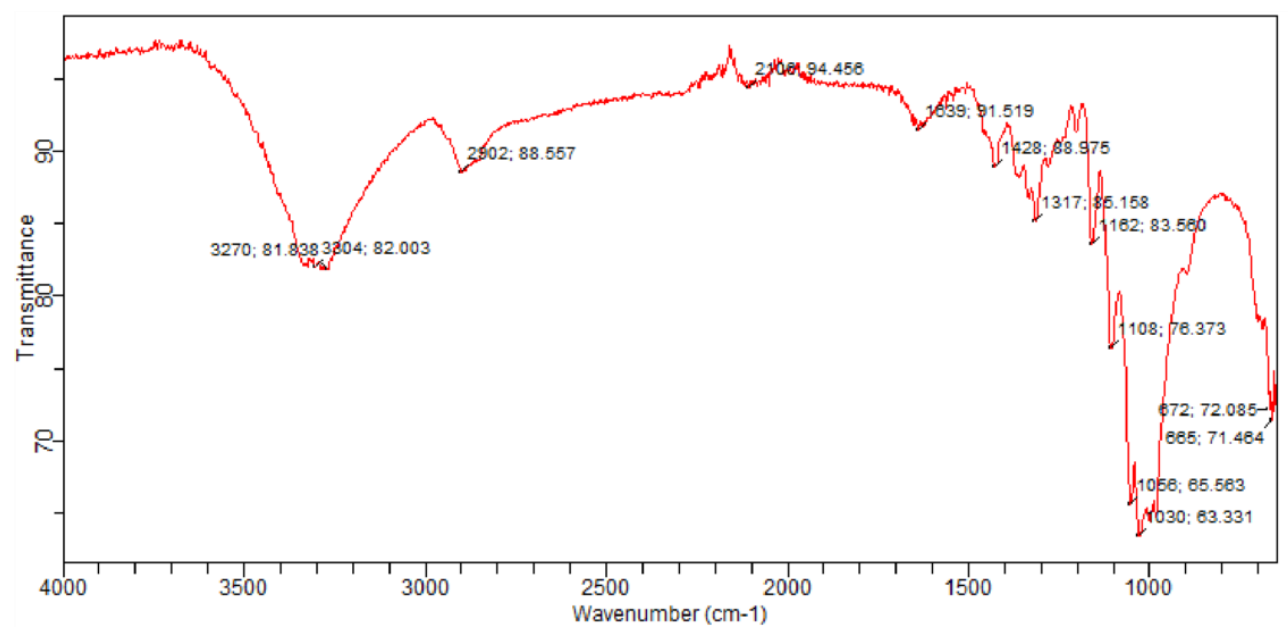

Figure 1c:FTIR spectrum of chitosan modified polylactic acid

From table 1 and Figure (1a, 1b, and 1c)It was established that absorption bands in the region of $3000-2800 \mathrm{~cm}^{-1}$ was due $\mathrm{OH}$ stretching and bending vibration in both chitosan, PLA and PLA-Ch nation composites. While absorption peaks at $2800-3000 \mathrm{~cm}^{-1}$ were due $\mathrm{CH}$ stretching and bending vibration in the glucose pyranose rings of both chitosan, PLA \& Ch-PLA nano composites. Whereas absorption bands at $1750-1760 \mathrm{C}$ was due to $\mathrm{C}=0$ stretching vibrations in both chitosan, PLA and the Ch-PL matrix. Another peak at $1428-1448$ was due to $\mathrm{CH}$-Bending vibration.

An absorption at $1554 \mathrm{~cm}^{-1}$ was due to $\mathrm{NH}$ stretching vibration in the $2^{0}$ amide confirming amidation reaction. 
BAJOPAS Volume 14 Number 1, June, 2021

Mechanical properties of polyethylene/PLA-Ch nanocomposites

Analysis of tensile strength and elongation at break of the nano composites films yield a very good results. The results are depicted in table 2.

Table 2: Tensile testing values for Ch-PL/polyethylene nano composites

\begin{tabular}{|c|c|c|c|c|c|c|c|c|c|c|c|c|c|c|c|}
\hline \multirow{2}{*}{ P.E (wt \%) } & ) & $\mathrm{m}$ & $\mathrm{p} \mid \mathrm{e}$ & $\mathrm{V} 0$ & $\mathrm{u}$ & $\mathrm{m}$ & $\mathrm{s}$ & & 0 & $0 \mathrm{~m}$ & 1 & $\underline{\alpha}$ & & - & \\
\hline & \multicolumn{15}{|c|}{ Ch-PLA (wt\%) Tensile strength (PMA) Elongation (\%) } \\
\hline 5 & 95 & i n & $100 \mathrm{~m} \mathrm{l}$ & 1 & 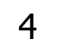 & 0 & & 9 & 6 & 0 & 3 & 2 & & 3 & 8 \\
\hline 1 & 90 & i n & & 1 & 8 & & & 2 & 0 & 0 & & & & & \\
\hline 5 & 85 & i $n$ & & 2 & 0 & c & & 6 & 1 & 0 & & 0 & & & \\
\hline 5 & 95 & i n & 200 & 2 & T & 0 & & 7 & 2 & 0 & 2 & 9 & & & \\
\hline 0 & 90 & i n & $200 \mathrm{~m} \mathrm{I}$ & 2 & 8 & 0 & & 0 & 0 & 6 & 2 & 9 & . & 0 & \\
\hline 5 & 5 & $n$ & & & 8 & 5 & & & 3 & & & & & 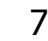 & \\
\hline
\end{tabular}

From table 2; it was observed that the tensile strength of the composites increases as a factor of polylactic-chitosan ratio, which indicates that the higher the increases in PLA-Ch concentration (\%) the higher the tensile strength and stiffer the composites films is.

Conversely, the elongation of the bioplastics to break decreases with increase in the concentration of Ch-PLA.

\section{Scanning Electron Microscopy of the Bioplastics}

The morphology of the Ch-PLA/polyethylene bioplastics showed a homogeneous surface independent of concentration of Ch-PLA or P.E in the composites.

The results may be attributed to the biocopatrbility of the Ch-PLA to the polyethylene in the composites. The results is in conformity with Nurshahida and Zainoha (2016). The micrographs of ChPLA, PE/Ch-PLA and chitosan were shown in Figure 1. 


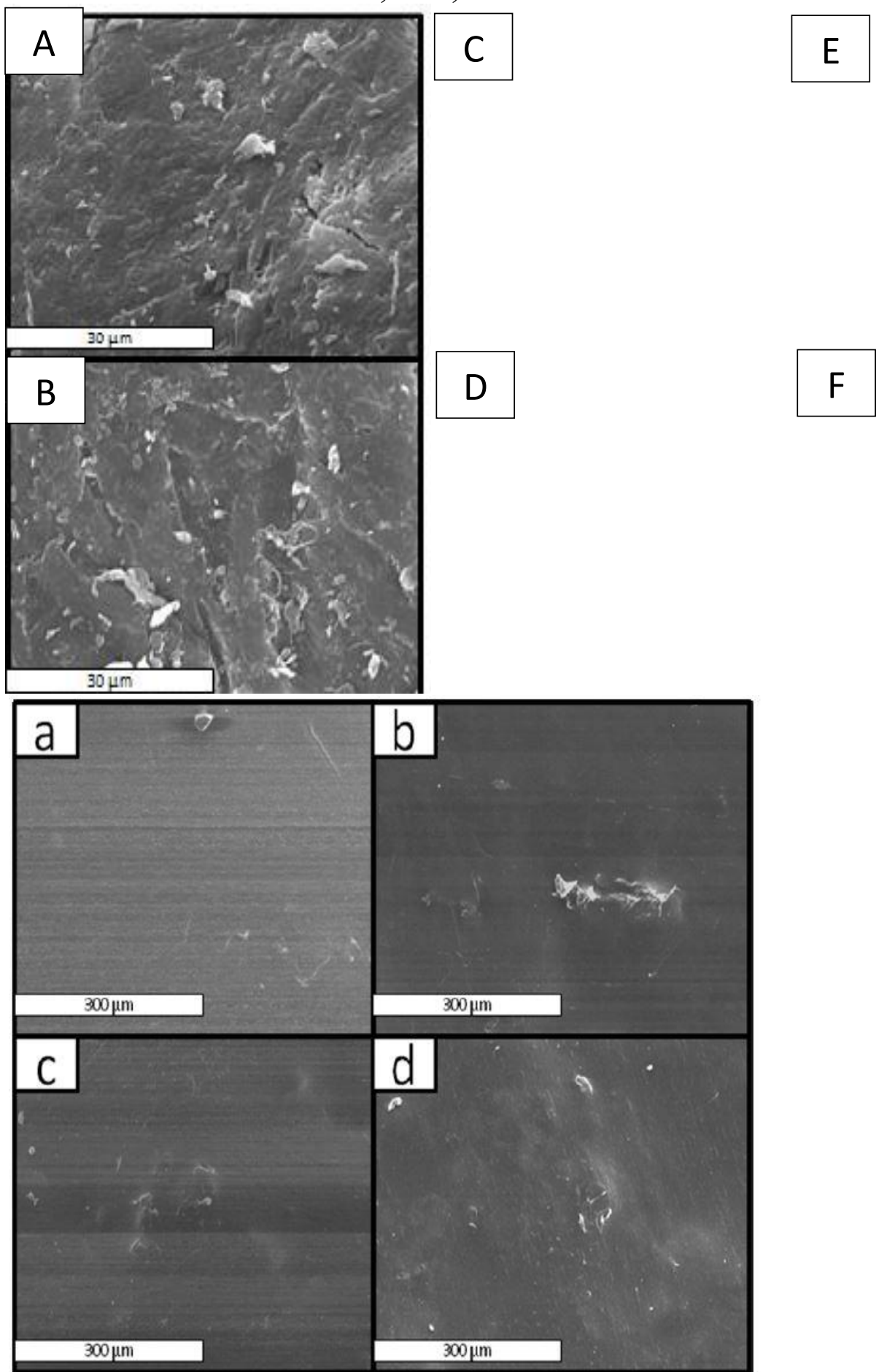

Figure 2: (a) Chitason (b) PLA (c) Ch-PLA (d) 5\% PE/Ch-PLA (e)10\% PE/Ch-PLA (f) 15\% PE/Ch-PLA

\section{Biodegradability of the bioplastics}

Biodegradation of the bioplastics were observed to be a factor of Ch-PLA concentrations. The results establish that biodegradation of the bioplastics occur effectively at concentration dependent manner of Ch-PLA content in both the samples as show in Figure 1. The results in agreement with Akbar et al., (2019). 

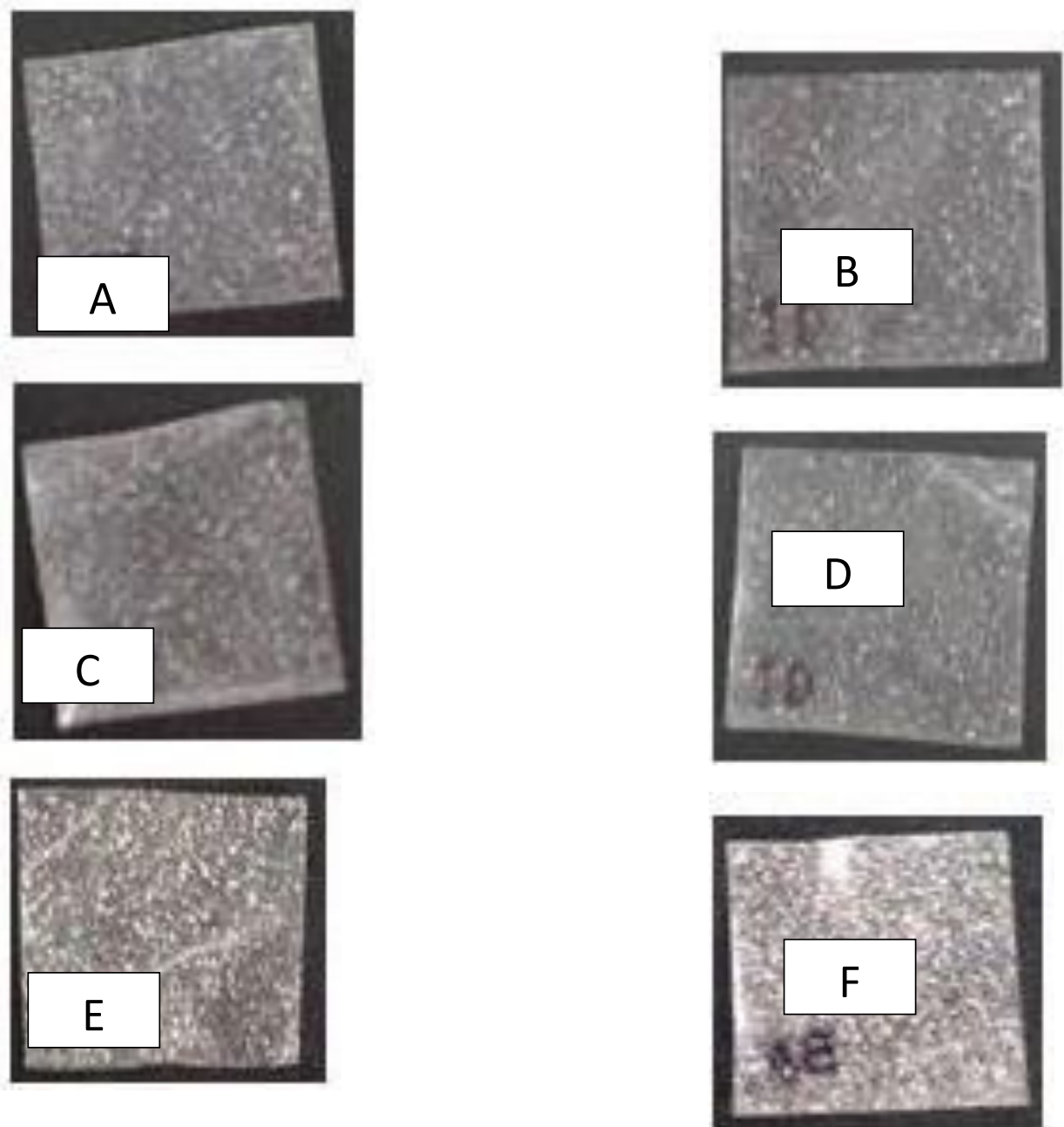

Figure 3: Biodegredable samples ( $a, c$ and e)Ch-PLA in $100 \mathrm{ml}(b, d$, and $f$ ) Ch-PLA in 200ml vol.

\section{CONCLUSION}

Biodegradable

polyethylene/biorenewable polymers composites were successfully prepared using grasshopper chitosan and potato waste (peel) based polylactic acid matrix. Concentration of Ch-PLA affect both physical and mechanical properties of the bioplastics composites by increasing the tensile strength in Ch-PLA concentration dependent manner. While the elongation to break of the nanocomposite was found to decrease in Ch-PLA content. The biodegradability of the composites was enhanced by enzymatic degradation in Ch-PLA dependent manner. The results indicated that it

\section{REFERENCES}

Akbar, H.A., Sri, P., Myrtha, K., Oceu, D.P. and Rani, H.F (2019). Fabrication and characterization of sweet potato starch based bioplastics plasticized with glycerol. Journal of biological sciences, 19(1): 57-64

Formin, V.A., Korovin, L.,P., Beloded, L.N., Kurski Shkurenko, S.I., Monakhora, E.V is very possible to manufacture films of synthetic polyethylene (P.E) with natural polymers Ch-PLA that is biodegradable as a means of utilization of potato waste and plastic waste in the industrial preparation of biodegradable plastics for food packaging.

\section{Acknowledgements}

The authors acknowledge the intellectual contribution and untiring efforts given by Prof. Haruna Musa Danbatta during the conduct of this research and when preparing this manuscript. Equally acknowledged the parental courage and support by Prof. A.A Salis that lead to the success of this research.

and Petrov, A.C (2017) investigating the process sof producing polilactic acid as the bse polymer of biodegred able polymers from renewable reosurces.

Polymers from From renewable Resources. 2( 1): $484-494$

Ke-ke, Y., Xiu-Li W.., and Yu-Zhong W. (2007) progress in Nanocomosites of 
BAJOPAS Volume 14 Number 1, June, 2021 biodegradable polymer J. Ind. Eng Chem 13(4): 485-500

Layla, B., S Joed, V.D., Veertede, G., Marijin T., Joe S., Carmen, B., Karim S. (2018) Addition of chitic nanoparticles myproves polylactic acid film properties nanotechnology and advanced materials sciences 1(2)1-8

Mohd Z.A., Nurhidayatullahi, M.J., Hafizan, J., Fazureen A. Nurhishaam, S., Ismail, Z.A (2015) fabrication and properties of chitosan with starch for packaging Application Malaysian journal of analytical sciences, 19(5): 1021-1042

Nurshalida, B.R and Zainoha Z. (2016) Biodegreability properties of chitin/polylactic acid composite filsm proceddings chemistry $1(2016) 48-52$ http://eproceedings.chemistry.utm.my/e ISSN2550-1453

Suryani, H., Agusnar, B., Wirjosentono T., Rihayat, Z., Salisah (2017). Synthesis and characterization of polylactic acid chitosan nanocomposites based on renewable resources and biobased materials. Second international joint conference of sciences and technology IOP conf. journal of physics conf. series 953(2018) 012015.

Tarek, A and Bader M.A. (2016) preparation, characterization and potential application of chitosan, chito san derivatives and chitosan nanoparticle sin pharmaceutical rug delivery (2016). Drug design, development and therapy 2016: (10): 483-507

Vincento, P. (2013) polylactic acid: synthesis, properties and applications. Nora science publishers inc. New York.

Yaret G.T., Gloria M.O., Lucia T.J., Nayelishanta I..J.Y Alejandro A.T., Blanca E.G. (2018). Biological compatability chitosan obtained from shrimp waste material 2018, 11, 1465, doi:3390/ma11061465 www.mdpi/com/jorunal/materails. 